\title{
CONTRIBUCIÓN DE LOS INDICADORES DE GESTIÓN EN LA EFICIENCIA ORGANIZACIONAL $\quad$ Y LA ADMINISTRACIÓN HOSPITALARIA EN INSTITUCIONES DE SALUD
}

\author{
AUTORES: Maylevis Morejón Valdés ${ }^{1}$ \\ José Felipe Ramírez Pérez ${ }^{2}$ \\ Eunice Vargas Contreras ${ }^{3}$ \\ Patricio Sebastian Henriquez Ritchie ${ }^{4}$
}

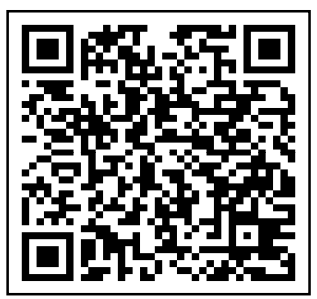

DIRECCIÓN PARA CORRESPONDENCIA: maylevis.morejon@uabc.edu.mx

Fecha de recepción: 01/02/2021

Fecha de aceptación: 28/06/2021

\section{RESUMEN}

En la actualidad la demanda de servicios de salud con mayor calidad y cobertura ha aumentado. Tal necesidad se ha debido al envejecimiento de la población, al aumento de las enfermedades crónicas, a la crisis económica mundial y recientemente a la pandemia COVID-19. En tal escenario, se requiere de una estructura de salud sólida para la gestión de los servicios de salud, que permita optimizar el empleo de recursos, y aumentar el rendimiento y la eficiencia. En el logro de dicho propósito, los indicadores de salud constituyen los índices que permiten conocer la realidad del estado de salud y de los recursos disponibles en determinada institución, para evaluar el impacto de las acciones realizadas y su proyección en el cumplimiento de los objetivos trazados. El objetivo de la investigación es analizar los indicadores de gestión hospitalaria, su contribución sobre la eficiencia organizacional y la administración hospitalaria en instituciones de salud. La investigación se enmarca a las instituciones de salud del nivel hospitalario. El estudio tiene un diseño no experimental, con enfoque cualitativo y alcance exploratorio. La bibliografía consultada se corresponde de manera consistente con los resultados obtenidos y con las afirmaciones realizadas. Los resultados proponen un marco conceptual donde los indicadores de gestión hospitalaria contribuyen de manera favorable con la eficiencia organizacional y la administración hospitalaria en instituciones de salud. Además, se constata un impacto positivo sobre la calidad de los procesos ejecutados, el desempeño intra-hospitalario, la competitividad inter-hospitalaria, la toma de decisiones y el fortalecimiento de la estructura organizacional.

\footnotetext{
${ }^{1}$ Ingeniera en Ciencias Informáticas. Facultad de Ciencias Administrativas y Sociales. Universidad Autónoma de Baja California, Unidad Valle Dorado. Ensenada, Baja California, México. E-mail: maylevis.morejon@uabc.edu.mx

${ }^{2}$ Ingeniero en Ciencias Informáticas. Facultad de Ciencias Administrativas y Sociales. Universidad Autónoma de Baja California, Unidad Valle Dorado. Ensenada, Baja California, México. E-mail: jramirez14@uabc.edu.mx

${ }^{3}$ Doctora en Ciencias Psicológicas. Facultad de Ciencias Administrativas y Sociales. Universidad Autónoma de Baja California, Unidad Valle Dorado. Ensenada, Baja California, México. E-mail: eunice.vargas@uabc.edu.mx

${ }^{4}$ Doctor en Ciencias de la Educación. Facultad de Ciencias Administrativas y Sociales. Universidad Autónoma de Baja California, Unidad Valle Dorado. Ensenada, Baja California, México. E-mail: phenriquez@uabc.edu.mx
} 
Valeria Pacheco Ochoa, Ana Lucía Jiménez Pérez, José Felipe Ramírez Pérez

PALABRAS CLAVE: administración hospitalaria; eficiencia organizacional; indicadores de salud; instituciones de salud; toma de decisiones.

\title{
CONTRIBUTION OF MANAGEMENT INDICATORS TO ORGANIZATIONAL EFFICIENCY AND HOSPITAL ADMINISTRATION IN HEALTHCARE INSTITUTIONS
}

\begin{abstract}
Currently, the demand for health services with greater quality and coverage has increased. This need is due to the aging of the population, the increase in chronic diseases, the global economic crisis and recently the COVID-19 pandemic. In such a scenario, a solid health structure for the management of health services is required to optimize the use of resources and increase performance and efficiency. In order to achieve this purpose, health indicators constitute the indexes that make it possible to know the reality of the state of health and the resources available in a given institution, to evaluate the impact of the actions carried out and their projection in the fulfillment of the objectives set. The aims is to analyze hospital management indicators and their contribution to organizational efficiency and hospital administration in health institutions. The research is framed to health institutions at the hospital level. The study has a non-experimental design, with a qualitative approach and exploratory scope. The bibliography consulted corresponds consistently with the results obtained and with the statements made. The results propose a conceptual framework in which hospital management indicators contribute favorably to organizational efficiency and hospital administration in health institutions. In addition, a positive impact on the quality of the processes executed, intra-hospital performance, inter-hospital competitiveness, decision making and strengthening of the organizational structure is observed.
\end{abstract}

KEYWORDS: decision making; health facilities; health status indicators; hospital administration; organizational efficiency.

\section{INTRODUCCIÓN}

La salud pública en su carácter universal tiene el propósito de alcanzar los más altos niveles de bienestar social, mental y físico, así como mayores niveles de longevidad poblacional. Actualmente, la demanda de servicios médicos ha aumentado considerablemente por diversos factores, en donde la pandemia COVID-19 ha tenido una elevada implicación desde finales de 2019. Es por ello que cada vez más los directivos en salud enfocan su atención en el desarrollo de acciones para lograr un empleo más eficiente de los recursos por su carácter limitado (Quintero, Ruiz, Martínez \& Rodríguez, 2017).

La administración en salud puede ser definida como una ciencia técnica y social en la que se planifican, organizan, dirigen y controlan las empresas privadas y públicas del sector de la salud (Livon, 2019; Rodríguez \& Salinas, 2017; Ugalde et al., 2018). Esta área de conocimiento tiene como funciones la coordinación y evaluación de los recursos y procedimientos, por los cuales la demanda de atención de salud y las necesidades de un ambiente sano son recibidas por la prestación de servicios a la población (Junior \& Porto, 2018). 
Los sistemas de salud están formados por la interconexión entre todas las organizaciones sanitarias, en la que cada una de sus partes requiere de administradores y los médicos son, en su mayoría, quienes realizan estas funciones. Es por ello que la administración en salud requiere de un continuo fortalecimiento y de fomentar la conciencia de su importancia. En este sentido, para que los sistemas de salud puedan operar como una inversión y no como un gasto, se necesitan resolver todos los problemas que están asociados con la adecuada implementación de estrategias, la gestión del conocimiento, la innovación, el empleo de las tecnologías de la información, la organización de los procesos sustantivos, así como la determinación de las competencias profesionales de su personal en cada una de sus áreas y departamentos, una toma de decisiones oportuna basada en datos, la descentralización de las funciones y la existencia de un buen liderazgo (Castell-FloritSerrate, 2017; Cristo, Pérez \& Izaguirre; 2020; Pérez \& Téllez, 2015; Zayas, Pérez \& Pérez, 2020).

Los hospitales, tanto públicos como privados, son uno de los componentes integrales de los sistemas de salud de los países, que requieren de una sólida capacidad de gestión y administración. Por lo tanto, el fortalecimiento de la capacidad de gestión dentro del sistema de salud, especialmente los hospitales, constituye una decisión estratégica para mejorar el desempeño de la prestación de servicios de salud y el sistema de salud en general (Rabbani et al., 2015). Para el logro de una adecuada gestión hospitalaria, los directivos y su estructura de dirección no basan sus decisiones en la suposición de que todo va e irá bien, en cambio, operan bajo la lógica de ser eficientes, productivos y eficaces en la prestación de servicios de calidad. Además, se ejecutan las funciones del proceso administrativo, que requiere una adecuada planificación, organización, dirección y control de las actividades y recursos existentes. Adicionalmente, una gestión hospitalaria ajustada a esos criterios considerará la necesidad de definir un conjunto de indicadores de gestión de las unidades de salud (González \& Barrios, 2007).

En línea con la idea anterior, el Ministerio de Salud del Perú ratifica que la gestión hospitalaria debe ser evaluada en el tiempo, por lo que es necesaria la aplicación de indicadores de gestión o indicadores en salud, como es definido este término en los descriptores de ciencias de la salud. Según Vílchez, Pardo \& Mendoza (2013) los indicadores de gestión se definen como la relación entre las variables cuantitativas y cualitativas, que permiten observar la situación y las tendencias de cambio generadas en una organización, de acuerdo con los objetivos definidos y metas esperadas. En cambio, para Grajales \& Hernández (2018) los indicadores de gestión son elementos para objetivar las políticas, planes y programas. Estos miden el impacto de las acciones, permiten racionalizar el gasto y son útiles para el control y la evaluación de objetivos y metas. Además, se emplean en el seguimiento y evolución de los bienes y servicios, y permiten la identificación de las áreas, de los puntos débiles y fuertes en la organización. Por su parte, González \& Barrios (2007) afirman que son necesarios para conocer el desempeño de las instituciones hospitalarias a partir de la información obtenida y constituyen un apoyo para la toma de decisiones. Su uso es de utilidad para las autoridades sanitarias y para todas las personas vinculadas con el perfeccionamiento del sector de la salud (Paneque, 2004).

En el caso de los servicios hospitalarios de calidad, el control de la gestión es fundamental para la eficacia y la eficiencia de la organización. Por lo tanto, se hace necesario que los gerentes puedan 
evaluar el desempeño de la organización, con el fin de medir su propia eficacia en la gestión (Junior \& Porto, 2018). En el boletín emitido por la Comisión Nacional de Arbitraje Médico (CONAMED) en el periodo comprendido de septiembre a octubre del 2018, se plantea que uno de los propósitos fundamentales de todo sistema de salud es asegurar la provisión de servicios equitativos, oportunos, efectivos y de calidad a la población. En México el Sistema Nacional de Salud vigente ha obtenido muy buenos logros en este sentido, los cuales se ven materializados en un aumento de la esperanza de vida, así como en la mejoría del estado de salud de su población. No obstante, teniendo en consideración todos sus logros, también constituye una realidad los retos que aún se tienen por delante. Uno de estos retos es el aumento de la eficiencia y el mejoramiento de los niveles de calidad, debido a la inequidad actual de los servicios de salud, cuya condición es esencial para asegurar el acceso efectivo a la atención para toda la población (Cantón, 2018).

Según Quintero et al. (2017), la eficiencia hospitalaria se define como la obtención de resultados satisfactorios de manera progresiva y sostenida, con un menor empleo de recursos, hacer más y mejor con menos. Es por ello que los autores refieren que para ello debe conocerse en detalle la organización, sus procesos y estrategias implementadas, para el logro adecuado de este propósito. La eficiencia guarda una relación directa con la eficacia y la efectividad (Rojas, Mesa \& Basulto, 2018). La efectividad es de mayor alcance y se define como la medida del impacto que las acciones realizadas tienen sobre la salud de la población. En el análisis de la eficacia se tienen en consideración los costos y los gastos asociados con la efectividad o la eficiencia que han sido alcanzados. Se trata de tres conceptos concatenados, pues no se concibe eficiencia sin efectividad y esta pierde sentido sin eficacia (Campanella et al., 2017; Paneque, 2004).

Todo lo anterior implica la necesidad de contar con información relevante, pertinente y confiable que permita la toma de decisiones oportuna y adecuada. En dicho sentido, las decisiones efectivas solo podrán tomarse a partir de un serio análisis de información, que implica la existencia de instrumentos adecuados de medición, incluidos una selección de indicadores en salud, orientados hacia la calidad de la atención médica (Cantón, 2018).

Las instituciones de salud del nivel hospitalario son centros complejos con disímiles tareas, actividades y procesos que son interdependientes entre sí. En este escenario es clave la gestión coordinada de actividades para ofrecer servicios de alta calidad con un presupuesto limitado (Naranjo-Gil, 2016). Hoy en día, entidades hospitalarias se ven afectadas por situaciones de cambios constantes y características muy distintas a las épocas anteriores. Ejemplo de ello lo constituye las modificaciones profundas en la estructura organizativa, nuevos sistemas de dirección, cultura de calidad y excelencia, reconocimiento de la responsabilidad hospitalaria, desarrollo de la innovación tecnológica e importancia del servicio al paciente (De La Cruz \& Claro, 2018).

Las razones de por qué y para qué se necesitan los indicadores son múltiples y muy diversas. Puede plantearse que en todos los casos su utilidad está ligada a los procesos de evaluación y monitoreo de proyectos, así como a la consecución de metas y el control de los procesos vinculados con la información que se pretende medir. Los indicadores cumplen con una función estratégica que provee información relevante para la conducción de política en salud como producto de la actuación coordinada y sinérgica de actividades de gestión de servicios de salud, vigilancia en salud pública e investigación epidemiológica. Su carácter estratégico se basa en la 
oportunidad y precisión de la información que se genera y en la combinación de sus capacidades diagnóstica y predictiva de la situación de salud (Cantón, 2018). Un mejor control de los indicadores permite optimizar y crear mecanismos permanentes de monitoreo respecto al avance, resultado y alcance del trabajo constante de la institución sanitaria. Es mediante los indicadores que se permite el cambio de un sistema de monitoreo tradicional por actividades, a un sistema de monitoreo de resultados. Su uso sistemático permite ubicar el nivel de eficacia y eficiencia de la organización, sin dar margen a la ambigüedad.

\section{ANTECEDENTES}

Los indicadores son una expresión cualitativa o cuantitativa observable. Brindan información relevante sobre las características y el desempeño de los servicios. Además, permiten comunicar y analizar información y comportamientos observables, a través de la evolución de una variable o el establecimiento de una relación entre variables (Camacho-Rui, Carrillo-Reyes, Rioja-Paradela \& Espinoza-Medinilla, 2016). Otras características que tienen los indicadores de salud son que permiten evaluar el desempeño y su evolución en el tiempo, realizar análisis de desviaciones de actividades, dar seguimiento a los objetivos planteados, así como procesar información para mejorar la toma de decisiones (Lima et al., 2016; Santana, Sarquis, Miranda, Kalinke, Felli \& Mininel, 2016). De igual manera los indicadores de salud favorecen la valoración del estado de salud actual de la entidad, el monitoreo del cumplimiento de acuerdos, la cuantificación de los cambios en una situación que se considera problemática, y dar seguimiento a los planes y programas, que permita tomar los correctivos oportunos y mejorar la eficiencia y eficacia del proceso en general (DANE, 2010; Wang, Tsui \& Miao, 2017). En la Figura 1 se representa la interrelación de los indicadores dado el nivel de resultados y jerarquía (DANE, 2010).

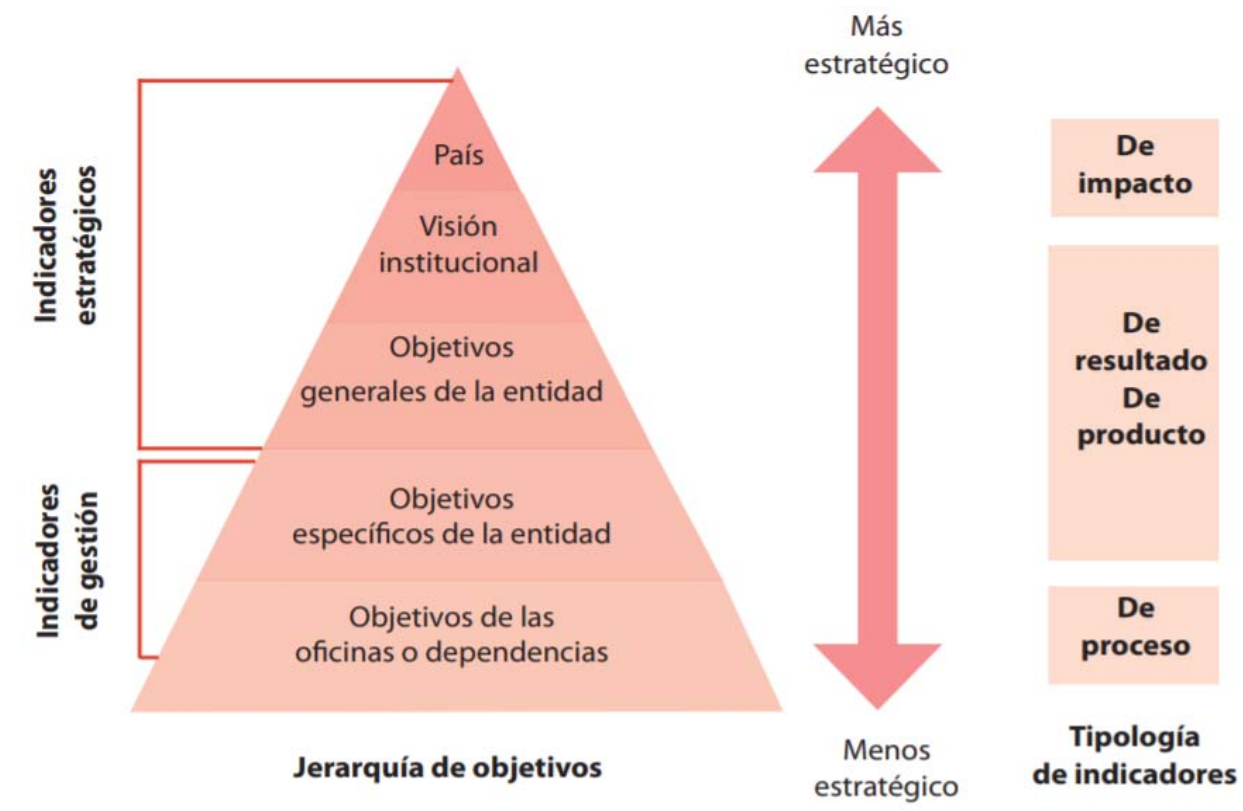

Figura 1. Interrelación entre indicadores, según nivel de resultados y jerarquía. Fuente: (DANE, 2010). 
Como ilustra la Figura 1, los indicadores de gestión constituyen la base de la pirámide. En la presente investigación se abordarán los indicadores de gestión para las entidades del sector de la salud, los cuales se consideran como instrumentos de medición que guían y apoyan el proceso de gestión asistencial y administrativa. La Secretaria de Salud de México tiene publicado un manual y una serie histórica de indicadores hospitalarios. La aplicación de los mismos tienda a ser homogénea en todas las instituciones del sector salud y en todas las entidades federativas del país (DGED, 2014). A pesar de que se encuentran definidos en el país, cada institución maneja sus propios indicadores de gestión, de manera que les permite un mejor control sobre la gestión de la entidad, así como apoyo a la toma de decisiones.

Teniendo en cuenta los aspectos abordados, el objetivo de la investigación es analizar los indicadores de gestión hospitalaria, así como su contribución sobre la eficiencia organizacional y la administración hospitalaria en instituciones de salud.

\section{MÉTODO}

La investigación enmarca su área de estudio a las instituciones de salud que brindan servicios en el segundo y tercer nivel de atención médica. El segundo nivel de atención se encuentra compuesto por hospitales generales y establecimientos que brindan los servicios de hospitalización y urgencias. Las principales especialidades que operan en el segundo nivel de atención son las de medicina interna, pediatría, ginecología, traumatología, urología y cirugía general. El tercer nivel de atención comprende hospitales especializados en una patología específica, por lo que la complejidad asistencial es elevada, es por ello que se requiere de conocimientos y estudios muy específicos.

El objetivo de la investigación es analizar los indicadores de gestión hospitalaria, así como su contribución sobre la eficiencia organizacional y la administración hospitalaria en instituciones de salud. Para el logro del objetivo definido se empleó un enfoque cualitativo, con diseño no experimental. Este tipo de estudios se llevan a cabo sin la manipulación de variables, por lo que no se efectúan proceso experimentales, sino que los procedimientos se concentran en observar la realidad analizada (Sampieri, Collado \& Lucio, 2014). En ella se analiza el impacto o la contribución que tienen los indicadores de gestión en la eficiencia organizacional y la administración hospitalaria en instituciones de salud. Las variables analizadas fueron los indicadores de gestión, la administración hospitalaria y la eficiencia organizacional.

Adicionalmente, es un estudio con alcance exploratorio. En este estudio se persigue tener una primera aproximación a los conceptos y aspectos asociados con los indicadores de gestión en instituciones de salud, en sus niveles secundario y terciario. Asimismo, se desea analizar el impacto o la contribución que tienen estos a la eficiencia organizacional y la administración hospitalaria. Para este fin se analizan y discuten las principales contribuciones realizadas por los autores especializados en estos temas, para arribar a conclusiones.

En la investigación se utilizan los siguientes métodos de investigación (Sampieri et al., 2014):

- Histórico-lógico: Fue empleado para analizar la evolución y desarrollo de la información y los conocimientos relativos con los indicadores de gestión y su incidencia cada vez mayor en la eficiencia organizacional y la administración hospitalaria. Mediante su aplicación se pudo resumir las principales

114 UNESUM-Ciencias. Publicación cuatrimestral. Vol. 5, Año 2021, No. 4 (Número Especial) 
contribuciones realizadas a nivel mundial por los investigadores en el área de conocimiento abordada.

- Analítico-sintético: Fue adoptado en el análisis concreto de los principales conceptos de la investigación, como son la administración hospitalaria, la eficiencia organizacional, los indicadores de salud, las instituciones de salud y la toma de decisiones. De esta manera se pudo abordar con referencias pertinentes y actualizadas cada temática y área de conocimiento de interés, yendo de lo general a lo particular.

- Análisis documental: Fue utilizado en el abordaje de los referentes teóricos de la investigación, así como en los conceptos antes mencionados, los cuales fueron los aspectos medulares del trabajo. Se realizó la consulta de artículos científicos en revistas digitales de impacto, así como en libros electrónicos. Las referencias empleadas tuvieron un 56\% de actualidad (2017-2021).

\section{RESULTADOS Y DISCUSIÓN}

De acuerdo con la literatura científica abordada, los resultados exploratorios que son presentados en la sección actual permiten analizar los indicadores de gestión hospitalaria, así como su contribución sobre la eficiencia organizacional y la administración hospitalaria en instituciones de salud. En la Figura 2 se visualizan las variables y dimensiones del fenómeno de estudio, soportadas en referencias actualizadas. Asimismo, tales hallazgos son discutidos como parte de la sección.

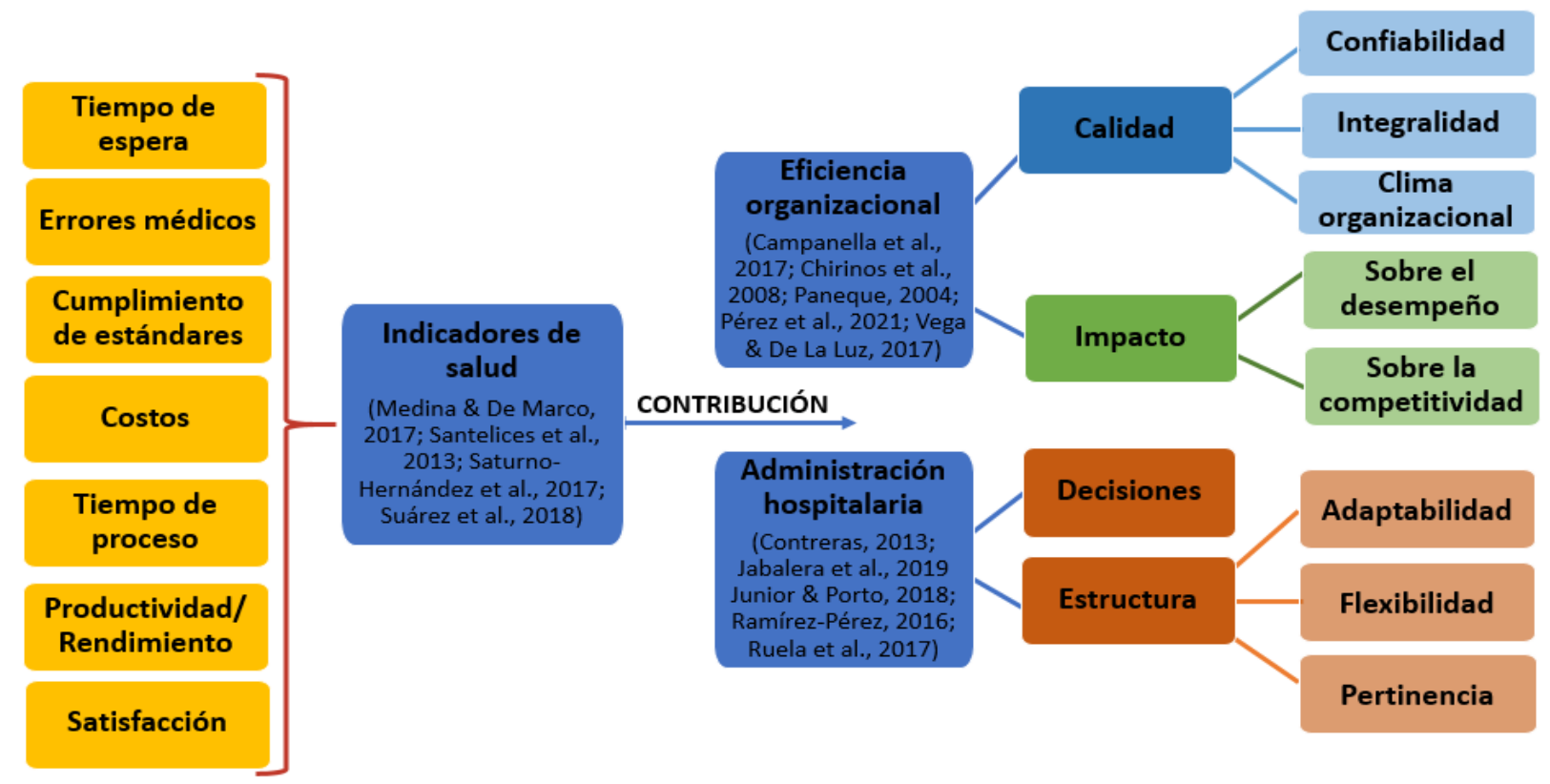

Figura 2. Marco conceptual sobre indicadores de salud y su contribución sobre la eficiencia organizacional y la administración hospitalaria. Fuente: elaboración propia. 
Los principales indicadores de salud en las instituciones hospitalarias se centran en obtener estadísticas respecto a los tiempos de espera (ejemplos: espera en admisión y en consulta eterna), los errores médicos (ejemplos: infecciones intrahospitalarias, suspensiones de cirugías y de reingresos), el cumplimiento de estándares (ejemplo: protocolos), los costos (ejemplos: turnos perdidos, y costo promedio por servicio), los tiempos de ejecución de los procesos sustantivos (ejemplos: atención médica y actividades de apoyo al diagnóstico), la productividad/rendimiento de la institución (ejemplos: rendimiento hora/médico e intervenciones por días/quirófanos) y la satisfacción del personal/pacientes/comunidad (ejemplos: quejas y reclamaciones).

Posteriormente, el resultado obtenido en cada uno de los indicadores de salud analizados va a contribuir a la eficiencia organizacional y a la administración hospitalaria. Tales afirmaciones, plasmadas en la Figura 2 se soportan en referencias actualizadas publicadas en revistas de alto impacto. La eficiencia organizacional puede ser medida desde la calidad en cuanto a la confiabilidad e integralidad de los procesos ejecutados, así como en el clima organizacional existente, que incluye a profesionales de la salud, pacientes, familiares y sociedad en sentido general. Del mismo modo, la eficiencia organizacional puede ser evaluada desde el impacto sobre el desempeño hacia el interior de la organización de salud y hacia el exterior, vista desde la competitividad inter-hospitalaria.

Adicionalmente, la contribución de los indicadores de salud a la administración hospitalaria puede ser evaluada desde el proceso de toma de decisiones oportuno y basado en la tenencia de datos, así como desde el fortalecimiento de la estructura organizacional, la cual va a alcanzar una mayor adaptabilidad y flexibilidad, así como mayor pertinencia desde el cumplimiento efectivo de los objetivos institucionales. Todo ello se debe a que una correcta gestión y toma de decisiones, basado en los indicadores de salud, le posibilitarán al hospital una mejor planificación, organización, dirección y control de las actividades y recursos, siendo más eficiente y sólida en su funcionamiento y previsión futura.

Con base en los resultados evidenciados, diversas investigaciones como las realizadas por Betancourt \& Sánchez (2015) afirman que la gestión de los indicadores tiene una elevada importancia, ya que se control permite a los directivos guiar, orientar y evaluar a la organización hacia sus objetivos estratégicos. Para Betancourt y Sánchez, difícilmente lo que no puede ser medido, no se va a poder controlar, como tampoco se va a poder mejorar. A tales resultados también arriban los autores de la presente investigación. Es por ello que De La Cruz \& Reyes (2017) y Rabbani et al. (2015) abordan que ejercer un liderazgo, a partir de la tenencia de datos originados de los indicadores de salud, permite la toma de decisiones estratégicas. De igual manera, posibilita la formulación de políticas y el establecimiento de estrategias adecuadas que vayan en correspondencia con los objetivos trazados por la organización de salud. Por todo lo anterior, resulta esencial el logro de un eficiente control interno de la ejecución de los procesos, y el empleo dado a los medios y recursos, desde los indicadores de salud, lo cual favorecerá también el enfrentamiento de los riesgos como consecuencia de los cambios en el contexto (Aguerrebere, 2012; Jabalera, Pons, Gómez \& Del Castillo, 2019; Queiroz, Albuquerque \& Malik, 2013).

Pérez-Romero, et al. (2017) realizaron un análisis de la eficiencia técnica y la productividad de los hospitales generales del Sistema Nacional de Salud español entre 2010 y 2012, con el fin de identificar variables hospitalarias y regionales explicativas. Para ello lo aplicaron a 230 hospitales 
por medio de un análisis envolvente de datos midiendo la eficiencia técnica global, pura, de escala y el índice de Malmquist. La robustez del análisis se evaluó con modelos input-output alternativos. Se emplearon modelos multinivel lineales transversales de efectos fijos para analizar las variables explicativas de eficiencia. Los resultados del análisis arrojaron que existe un amplio margen de mejora en la eficiencia hospitalaria. Se señala y cuantifica la importancia de las características sociodemográficas y de las políticas de gasto público regional para explicar la eficiencia del hospital. Aporta a la investigación el conocimiento de que la eficiencia hospitalaria depende de las características de cada entidad de salud.

Además, Castelli et al. (2015) plantea que en muchos artículos la eficiencia hospitalaria se mide, principalmente, utilizando una técnica conocida como análisis envolvente de datos. La técnica puede acomodar el análisis de múltiples salidas y entradas; lo hace aplicando programación lineal para buscar un conjunto de pesos específicos de la organización con los que combinar diversas salidas en una única función (y lo mismo para las entradas). Dicha técnica tiene como deficiencia que el número de salidas de cada hospital generalmente supera al número de hospitales. Es por ello que los autores proponen un enfoque alternativo, que implica el uso de ponderaciones explícitas para combinar diversos resultados en un solo índice, evitando así la necesidad del análisis envolvente de datos. Su interés es medir la productividad relativa entre hospitales y la definen como como la relación entre productos e insumos. Para ello construyeron un rango de variables sobre la actividad de los pacientes hospitalizados utilizando datos extraídos de la base de datos de estadísticas de episodios hospitalarios y las asistencias de pacientes ambulatorios de la base de datos de costos de referencia.

Los autores Castelli et al. (2015) construyeron medidas de productividad para cada hospital del NHS (Servicio Nacional de Salud de Inglaterra, por sus siglas en inglés) y analizaron por qué la productividad varía de un hospital a otro especificando modelos econométricos en los que se hace una regresión de la productividad frente a una variedad de variables que capturan las características de cada hospital. Examinan las variaciones de la productividad hospitalaria y consideran dos variables que capturan la eficiencia en el uso de recursos. Estas son la proporción de camas ocupadas sobre el total de camas y la duración promedio de la estadía, que se calcula como la relación entre el total de días de hospitalización y el número total de pacientes. Los autores también encontraron que la productividad es mayor en los hospitales con mayor autonomía financiera, y donde una gran proporción de los ingresos proviene de actividades de educación, investigación, desarrollo y capacitación.

En México, Moreno-Martínez y Martínez-Cruz (2015) realizaron un estudio que tiene el objetivo de determinar la eficiencia hospitalaria a través del recurso cama en un hospital de segundo nivel. Para ello trabajaron con el promedio de días estancia (PDE) y el porcentaje de ocupación hospitalaria en unidades de segundo nivel (PO). Tales indicadores son señalados como estratégicos en el Manual Metodológico 2013 del Instituto Mexicano del Seguro Social (IMSS). Además, trabajaron con el índice de rotación de camas (IRC), el intervalo de sustitución de camas (ISC) y el índice de camas censables por 1000 habitantes. En este caso, estos indicadores son utilizados por la Secretaria de Salud, considerados igualmente muy importantes.

El estudio tuvo un diseño ecológico exploratorio. Tuvo como unidad de análisis la cama hospitalaria. Se examinaron, del Sistema de Información Médico Operativo (SIMO), los cuadros 
de salida mensuales de julio 2012 a junio 2013 del Hospital General con Medicina Familiar 1 Tapachula (HGZMF 1) que incluyen indicadores como egresos, días pacientes, porcentaje de ocupación y promedio de días de estancia por especialidad y división. Se obtuvieron los resultados por cada indicador estratégico y se relacionaron dichos resultados proponiendo supuestos para valorar la eficiencia hospitalaria. Para medir la eficiencia hospitalaria se relacionó y analizó la variación que existe entre los indicadores. Se propuso en el estudio cuatro niveles de eficiencia: deteriorada, baja, media y óptima. Se identificaron servicios con indicadores que señalan la prolongada estadía de los pacientes al presentar más días hospitalizados que los esperados, así como una subutilización de en algunos servicios.

Para la presente investigación se toman en cuenta los siguientes elementos del estudio realizado por Moreno-Martínez y Martínez-Cruz (2015):

- Los indicadores relacionados con la hospitalización de los pacientes son muy importantes para evaluar eficiencia hospitalaria, principalmente la estadía y la ocupación hospitalaria.

- Además de los indicadores mencionados, para abordar eficiencia, se debe tener en cuenta la estructura, el proceso y el resultado, desde la perspectiva de calidad de los servicios de salud. Esto lo presentan como limitante en el estudio pero resaltan que es un elemento importante a tener en cuenta.

- Plantean que en años recientes las estrategias para disminuir el gasto hospitalario se han basado en la reducción de camas hospitalarias, disminución de las estancias y reducción del número de ingresos. Proponen alternativas a esta problemática: la reorganización física de los servicios y ajustar el indicador por tipo de pacientes atendidos en los servicios, por medio de algún sistema de clasificación de pacientes, como los grupos de diagnósticos relacionados.

- Se emplea un semáforo de colores correspondiente a los cuatro niveles de eficiencia definidos.

- Los nuevos instrumentos de gestión deben ir encaminados a intentar predecir los niveles de ocupación de camas, para hacer un uso más eficiente de las mismas.

La principal limitante del estudio realizado surge del alcance de la investigación, la cual al ser exploratoria solo identifica, propone y discute un marco conceptual para el análisis de los indicadores de salud considerados más importantes en las instituciones de salud del nivel hospitalario. Para ello se tiene en cuenta un enfoque genérico en el análisis de los indicadores, debido a que cada institución de salud es distinta y en muchas ocasiones implementa nuevos indicadores para el análisis de cuestiones particulares consideradas igualmente relevantes en su gestión y contexto. Por todo ello, se deben realizar intervenciones que analicen concretamente la contribución que tienen los indicadores de gestión hospitalaria sobre la eficiencia organizacional y la administración hospitalaria en instituciones de salud, así como en cada uno de los departamentos y servicios hospitalarios. 


\section{CONCLUSIONES}

Luego de realizada la investigación se concluye que los indicadores de gestión hospitalaria contribuyen con la eficiencia organizacional y la administración hospitalaria en instituciones de salud. La bibliografía consultada se corresponde de manera consistente con los resultados obtenidos y con las afirmaciones realizadas. Por todo ello se propone un marco conceptual que aborda las tres variables (indicadores de salud, eficiencia organizacional y administración hospitalaria), así como las dimensiones e indicadores que materializan y facilitan la percepción de su impacto. Como principales indicadores de salud se encuentran los asociados con los tiempos de espera, los errores médicos, el cumplimiento de estándares, los costos, los tiempos de los procesos sustantivos, productividad/rendimiento y satisfacción. Del mismo modo, la eficiencia organizacional es evaluada desde las dimensiones Calidad e Impacto, mientras que la administración hospitalaria es medida desde las dimensiones Decisiones y Estructura,

Los trabajos futuros relacionados con la gestión de indicadores de gestión hospitalaria en las instituciones de salud deben dirigirse a la generación de investigaciones con alcances descriptivos, correlacionales o explicativos, así como con diseño experimental, las cuales analicen el efecto producido por una adecuada gestión de los indicadores en salud, sobre la eficiencia organizacional y la administración hospitalaria.

\section{REFERENCIAS BIBLIOGRÁFICAS}

Aguerrebere, P. M. (2012). El valor estratégico de la comunicación interna hospitalaria. Revista de comunicación y salud, 2(1), 19-28.

Camacho-Rui, E., Carrillo-Reyes, A., Rioja-Paradela, T. M., \& Espinoza-Medinilla, E. E. (2016). Indicadores de sostenibilidad para el ecoturismo en México: estado actual. LiminaR, 14(1), 156-168.

Campanella, P., Azzolini, E., Izzi, A., Pelone, F., De Meo, C., La Milia, D., ... \& Ricciardi, W. (2017). Hospital efficiency: how to spend less maintaining quality?. Annali dell'Istituto superiore di sanita, 53(1), 46-53.

Cantón, S. B. F. (2018). Los Indicadores como herramienta inteligente para la gestión de la calidad de los servicios de salud en México. BOLETÍN CONAMED, 4(20).

Castell-FloritSerrate, P. (2017). La administración en salud, componente de desarrollo de la salud pública. Revista Cubana de Salud Pública, 43(1), 1-2.

Chirinos, E., Rivero, E., Goyo, A., Méndez, E., \& Figueredo, C. (2008). Indicadores de gestión para medir la eficiencia hospitalaria. Negotium, 4(10), 50-63.

Cristo, N. M., Pérez, J. F. R., \& Izaguirre, L. V. (2020). Estrategia de entrenamiento y acompañamiento a usuarios para el Sistema de Información Hospitalaria XAVIA HIS. Revista Cubana de Informática Médica, 12(1), 7691.

DANE. (2010). Departamento Administrativo Nacional de Estadística (DANE). Guía para Diseño, Construcción e Interpretación de Indicadores. Estrategia para el Fortalecimiento Estadístico Territorial. Herramientas estadísticas para una gestión territorial más efectiva. Recuperado de

http://www.dane.gov.co/files/planificacion/fortalecimiento/cuadernillo/Guia_construccion_interpretacion_ind icadores.pdf

De La Cruz, L. O. V., \& Claro, I. I. T. (2018). Contribución a la implantación del control interno a través de sus variables activas en una entidad hospitalaria. Revista Médica Electrónica, 40(1), 13-24. ISSN 1684-1824

De La Cruz, L. O. V., \& Reyes, L. L. G. (2017). Diagnóstico estadístico del control interno en una institución hospitalaria. Revista Habanera de Ciencias Médicas, 16(2), 295-309. 
Valeria Pacheco Ochoa, Ana Lucía Jiménez Pérez, José Felipe Ramírez Pérez

DGED. (2014). Manual de Indicadores para Evaluación de Servicios Hospitalarios. Dirección General de Evaluación del Desempeño (DGED). Secretaría de Salud de México. Recuperado de http://www.dged.salud.gob.mx/contenidos/dess/ind_hospitalarios.html

González, V. V., \& Barrios, E. H. (2007). Indicadores de gestión hospitalaria. Revista de Ciencias Sociales (Ve), 13(3), 444-454.

Grajales, D., \& Hernández, H. H. G. (2018). Indicadores de gestión para los entes estatales del deporte, la recreación, el ocio y afines. Medellín: Intempo.

Jabalera, M., Pons, M., Gómez, E., Del Castillo, M., \& Grupo de Trabajo, E. M. C. (2019). Hacia la excelencia en gestión hospitalaria. Un modelo de gestión estratégica. Journal of Healthcare Quality Research, 34(3), 148153.

Junior, R. F., \& Porto, A. P. (2018). La calidad de vida en el trabajo y el ausentismo como indicadores de resultado de gestión hospitalaria. Ciencias Administrativas, 6.

Lima, V. A. D., Morosini, M. C., Fernandes, C. M. B., Leite, D., Franco, M. E. D. P., Cunha, M. I. D., y Isaia, S. M. A. (2016). A qualidade da educação superior e o complexo exercício de propor indicadores. Revista brasileira de educação. 21(64), 13-37.

Livon, F. O. (2019). Administración de salud pública y gestión de recursos humanos en la Microred Centenario de Abancay-2018. (Tesis doctoral). Universidad Cesar Vallejo. Perú. Recuperado de http://repositorio.ucv.edu.pe/handle/UCV/39013

Medina, O. F., \& De Marco, M. L. C. (2017). Indicadores de Productividad en Hospitales Públicos. Revista Científica "Visión de Futuro", 21(2), 40-60.

Naranjo-Gil, D. (2016). Cómo los equipos de dirección usan los sistemas de información y control en la gestión hospitalaria. Gaceta Sanitaria, 30(4), 287-292.

Paneque, R. E. J. (2004). Indicadores de calidad y eficiencia de los servicios hospitalarios: Una mirada actual. Revista Cubana de Salud Pública, 30(1), 0-0.

Pérez, J. F. R., López-Torres, V. G., \& Valdés, M. M. (2021). Information and Communication Technologies as a competitive performance factor in provider institutions of medical services in Ensenada, Baja California. Journal of Administrative Science, 2(4), 31-37.

Pérez-Romero, C., Ortega-Díaz, M. I., Ocaña-Riola, R., \& Martín-Martín, J. J. (2017). Análisis de la eficiencia técnica en los hospitales del Sistema Nacional de Salud español. Gaceta Sanitaria, 31, 108-115.

Pérez, J. F. R., \& Téllez, R. B. (2015). Propuesta de Red Cubana Aurora para la colaboración médica a través de Infomed utilizando un enfoque de redes sociales. In Convención Internacional de Salud, Cuba Salud 2015. La Habana, Cuba. Recuperado de

http://www.convencionsalud2015.sld.cu/index.php/convencionsalud/2015/paper/view/1649/658

Queiroz, A. C. S., Albuquerque, L. G. D., \& Malik, A. M. (2013). Gestión estratégica y innovación: estudio de caso en el contexto hospitalario. Revista de Administração (São Paulo), 48(4), 658-670.

Quintero, R. S. G., Ruiz, R. L., Martínez, L. A. B., \& Rodríguez, I. G. (2017). Aspectos teóricos sobre eficacia, efectividad y eficiencia en los servicios de salud. Revista información científica, 96(6), 1153-1163.

Rabbani, F., Hashmani, F. N., Mukhi, A. A. A., Gul, X., Pradhan, N., Hatcher, P., Farag, M., y Abbas, F. (2015). Hospital management training for the Eastern Mediterranean Region: time for a change? Journal of Health, Organization and Management, 29(7), 965-972.

Ramírez-Pérez, J. F. (2016). Modelo para la selección de equipos de trabajo quirúrgico en sistemas de información en salud aplicando técnicas de inteligencia organizacional. Editorial Universitaria. Recuperado de https://repositorio.uci.cu/handle/123456789/7161

Rodríguez, R. D. L. C. P., \& Salinas, A. M. (2017). La Administración de Salud: una materia urgida de trascendencia en la educación médica. EDUMECENTRO, 9(3), 312-315.

120 UNESUM-Ciencias. Publicación cuatrimestral. Vol. 5, Año 2021, No. 4 (Número Especial) 
Rojas, J. C., Mesa, A. P., \& Basulto, M. S. G. (2018). Enfoques teóricos para la evaluación de la eficiencia y eficacia en el primer nivel de atención médica de los servicios de salud del sector público. Retos de la Dirección, 12(1), 96-118.

Ruela, J. P. B., Aristega, J. E. M., Masacon, M. R. H., Huilcapi, G. V. B., \& Silvera, F. A. G. (2017). Costos operativos en la gestión administrativa y financiera en unidades hospitalarias. Pro Sciences: Revista de Producción, Ciencias e Investigación, 1(5), 22-27.

Sampieri, R. H., Collado, C. F., \& Lucio, P. B. (2014). Metodología de la Investigación, Sexta Edición México. DF, Editores, SA de CV.

Santana, L. D. L., Sarquis, L. M. M., Miranda, F. M. D. A., Kalinke, L. P., Felli, V. E. A., \& Mininel, V. A. (2016). Health indicators of workers of the hospital area. Revista brasileira de enfermagem, 69(1), 23-32.

Santelices, E., Ormeño, H., Delgado, M., Lui, C., Valdés, R., \& Durán, L. (2013). Análisis de los determinantes de la eficiencia hospitalaria: el caso de Chile. Revista Médica de Chile, 141(4), 457-463.

Saturno-Hernández, P. J., Martínez-Nicolás, I., Poblano-Verástegui, O., Vértiz-Ramírez, J. D. J., Suárez-Ortiz, E. C., Magaña-Izquierdo, M., \& Kawa-Karasik, S. (2017). Implementación de indicadores de calidad de la atención en hospitales públicos de tercer nivel en México. Salud Pública de México, 59, 227-235.

Suárez, L. M. C., Vivanco, A. S., del Arco, E. A., \& Martínez, A. M. (2018). Indicadores para mejorar la atención a pacientes según lean-seis-sigma: el caso del hospital Gustavo Fricke (Chile). Gerencia y Políticas de Salud, 17(35).

Ugalde, M. A. C., López, D. D., Quiroz, J. M. G., Tóala, S. J. M., Sánchez, L. M. P., y Quimis, T. L. M. (2018). La administración de la salud y la calidad del desempeño para los beneficiarios. Revista Científica de la Investigación y el Conocimiento, 2(4), 160-188.

Vílchez, W. C., Pardo, K., \& Mendoza, L. (2013). Indicadores de gestión y evaluación hospitalaria para hospitales, institutos y DIRESA. Ministerio de Salud, Perú.

Wang, D., Tsui, K. L., \& Miao, Q. (2017). Prognostics and health management: A review of vibration based bearing and gear health indicators. IEEE Access, 6, 665-676.

Zayas, J. C. B., Pérez, J. F. R., \& Pérez, A. D. R. R. (2020). Interacciones medicamentosas como un problema de salud imperceptible en la población. Revista Cubana de Medicina General Integral, 36(1), 1-15. 
122 UNESUM-Ciencias. Publicación cuatrimestral. Vol. 5, Año 2021, No. 4 (Número Especial) 